\title{
Density functional scheme for calculating the pair density
}

\author{
Masahiko Higuchi \\ Department of Physics, Faculty of Science, Shinshu University, Matsumoto 390-8621, Japan \\ Katsuhiko Higuchi \\ Graduate School of Advanced Sciences of Matter, \\ Hiroshima University, Higashi-Hiroshima 739-8527, Japan
}

(Dated: March 27, 2018)

\begin{abstract}
The density functional scheme for calculating the pair density is presented by means of the constrained-search technique. The resultant single-particle equation takes the form of the modified Hartree-Fock equation which contains the kinetic contribution of the exchange-correlation energy functional as the correlation potential. The practical form of the kinetic contribution is also proposed with the aid of the scaling relations of the kinetic energy functionals.
\end{abstract}

The pair density (PD) functional theory is expected to improve upon the conventional density functional theory [1, 2], because the PD has a larger amount of information than the electron density [3, 4, 5]. Recently the PD functional theory has been developed by several workers. Ziesche first proposed the PD functional theory using the natural spin geminals 6, 7]. Another scheme was offered by Gonis with taking the two-particle densities as a basic variable 8, 9]. Nagy generalized Gonis's theory to the PD functional theory which has the auxiliary equation of a two-particle problem [10, 11]. Ayers presented the well-conceived approximate form of the kinetic energy functional within the PD functional theory 12 .

In this paper, we propose the other type of the PD functional theory. Our strategy is to reproduce the PD by using the single Slater determinant (SSD). This strategy has two kinds of merits. The first merit is that the reproduced PD is never unphysical. This is a strong merit because the $N$-representability of the PD has been an unresolved problem, as yet 4, 13, 14, 15, 16, 17, 18, 19]. The details will be discussed later. The second merit is the interesting form of the single-particle equation. As shown later, it is the modified Hartree-Fock equation which additionally contains the kinetic contribution of the exchange-correlation energy functional as the correlation term. This form is reasonably intelligible to us because correlation effects are explicitly incorporated into the single-particle equation as the correction to the Hartree-Fock equation. Hereafter, we shall describe our scheme and the above merits in detail.

Let us begin with the Hohenberg-Kohn theorems with taking the PD as a basic variable. The PD is defined by

$$
\gamma^{(2)}\left(\mathbf{r r}^{\prime} ; \mathbf{r r}^{\prime}\right)=\left\langle\Psi\left|\frac{1}{2} \iint \hat{\psi}^{+}(x) \hat{\psi}^{+}\left(x^{\prime}\right) \hat{\psi}\left(x^{\prime}\right) \hat{\psi}(x) \mathrm{d} \eta \mathrm{d} \eta^{\prime}\right| \Psi\right\rangle,
$$

where $\hat{\psi}(x)$ and $\hat{\psi}^{+}(x)$ are field operators of electrons, $\Psi$ is the anitisymmetric wave function, and $x$ denotes the coordinates including the spatial coordinate $\mathbf{r}$ and spin coordinate $\eta$. We consider a system, the Hamiltonian of which is given by

$$
\hat{H}=\hat{T}+\hat{W}+\int \hat{\rho}(\mathbf{r}) v_{\text {ext }}(\mathbf{r}) \mathrm{d} \mathbf{r},
$$

where $\hat{T}, \hat{W}$ and $\hat{\rho}(\mathbf{r})$ are operators of the kinetic energy, electron-electron interaction and electron density, respectively, and $v_{\text {ext }}(\mathbf{r})$ stands for the external potential. The proof procedure is similar to the constrained-search theories 20, 21, 22, 23]. The universal functional $F\left[\gamma^{(2)}\right]$ is defined by

$$
\begin{aligned}
F\left[\gamma^{(2)}\right] & =\operatorname{Min}_{\Psi \rightarrow \gamma^{(2)}}\langle\Psi|\hat{T}+\hat{W}| \Psi\rangle \\
& =\left\langle\Psi\left[\gamma^{(2)}\right]|\hat{T}+\hat{W}| \Psi\left[\gamma^{(2)}\right]\right\rangle
\end{aligned}
$$

where $\Psi \rightarrow \gamma^{(2)}$ denotes the searching over all anitisymmetric wave functions that yield a particular $\gamma^{(2)}\left(\mathbf{r r}^{\prime} ; \mathbf{r r}^{\prime}\right)$. In the second line, the minimizing wave function is expressed as $\Psi\left[\gamma^{(2)}\right]$. Here we suppose that the minimum exists in Eq. (3). By virtue of the Rayleigh-Ritz principle, we get [6, 7]

$$
\Psi\left[\gamma_{0}^{(2)}\right]=\Psi_{0},
$$

where $\Psi_{0}$ is the ground-state wave function, and $\gamma_{0}^{(2)}\left(\mathbf{r r}^{\prime} ; \mathbf{r r}^{\prime}\right)$ is the ground-state PD given by $\gamma_{0}^{(2)}\left(\mathbf{r r}^{\prime} ; \mathbf{r r}^{\prime}\right)=$ $\left\langle\Psi_{0}\left|\hat{\gamma}^{(2)}\left(\mathbf{r r}^{\prime} ; \mathbf{r r}^{\prime}\right)\right| \Psi_{0}\right\rangle$. 
The variational principle with respect to $\gamma^{(2)}\left(\mathbf{r r}^{\prime} ; \mathbf{r r}^{\prime}\right)$ can be obtained by dividing the ordinary Rayleigh-Ritz principle into two steps: $E_{0}=\operatorname{Min}_{\gamma^{(2)}}\left\{\operatorname{Min}_{\Psi \rightarrow \gamma^{(2)}}\langle\Psi|\hat{H}| \Psi\rangle\right\}$. Using Eqs. (2) and (3), and defining the energy functional $E\left[\gamma^{(2)}\right]$ as

$$
E\left[\gamma^{(2)}\right] \equiv F\left[\gamma^{(2)}\right]+\frac{2}{N-1} \iint \gamma^{(2)}\left(\mathbf{r r}^{\prime} ; \mathbf{r r}^{\prime}\right) v_{\mathrm{ext}}(\mathbf{r}) \mathrm{d} \mathbf{r} \mathrm{d} \mathbf{r}^{\prime}
$$

the Rayleigh-Ritz principle is further rewritten as [6, 7]

$$
\begin{aligned}
E_{0} & =\underset{\gamma^{(2)}}{\operatorname{Min}} E\left[\gamma^{(2)}\right] \\
& =E\left[\gamma_{0}^{(2)}\right] .
\end{aligned}
$$

Equations (44) and (6) are regarded as the extened Hohenberg-Kohn theorems.

In order to perform the variational principle Eq. (6), it is necessary to restrict the searching area within the set of $N$-representable PD's. Only the necessary condition for the $N$-representable PD, which is called Pauli condition, has been realized by Coleman 13 . However, the necessary and sufficient condition for the $N$-representable PD is, as yet, the unresolved problem 4, 13, 14, 15, 16, 17, 18, 19. Our strategy is to restrict the searching area within not the set of $N$-representable PD's but that of PD's which are given by SSD's. If the former and latter sets are respectively denoted as $C$ and $C^{\prime}$, then $C \supseteq C^{\prime}$ holds obviously. Therefore, this strategy prevents the minimizing PD from being unphysical one. There is a possibility that the ground-state PD may belong to $C \cap \bar{C}^{\prime}$, where $\bar{C}^{\prime}$ stands for the complementary set of $C^{\prime}$. However, our strategy is to search the best solution within $C^{\prime}$. Best solution means the most appropriate reproduction of the PD with respect to searching the minimum of Eq. (6) within $C^{\prime}$. This spirit is analogous to the usual Hartree-Fock approximation because the best antisymmetric wave function is searched within the set of SSD's 24. Along this spirit, we shall give the practical and useful scheme for calculating the PD.

In order to perform the variational principle practically, we shall introduce the kinetic energy functional $T_{s}\left[\gamma^{(2)}\right]$ which is defined by

$$
T_{s}\left[\gamma^{(2)}\right] \equiv \operatorname{Min}_{\Phi \rightarrow \gamma^{(2)}}\langle\Phi|\hat{T}| \Phi\rangle
$$

where $\Phi$ is the SSD which is constructed from $N$ orthonormal spin orbitals $\psi_{\mu}(x)$, and $\Phi \rightarrow \gamma^{(2)}$ indicates that the search is constrained among all SSD's which yield the prescribed $\gamma^{(2)}\left(\mathbf{r r}^{\prime} ; \mathbf{r r}^{\prime}\right)$. We again suppose that the minimum exists in Eq. (7) similarly to Eq. (3). In Eq. (7), $\gamma^{(2)}\left(\mathbf{r r}^{\prime} ; \mathbf{r r}^{\prime}\right)$ is expressed as the expectation value with respect to the SSD. Using Eq. (11), we have

$$
\gamma^{(2)}\left(\mathbf{r r}^{\prime} ; \mathbf{r r}^{\prime}\right)=\frac{1}{2} \sum_{\mu, \nu=1}^{N} \iint\left\{\psi_{\mu}^{*}(x) \psi_{\nu}^{*}\left(x^{\prime}\right) \psi_{\mu}(x) \psi_{\nu}\left(x^{\prime}\right)-\psi_{\mu}^{*}(x) \psi_{\nu}^{*}\left(x^{\prime}\right) \psi_{\nu}(x) \psi_{\mu}\left(x^{\prime}\right)\right\} \mathrm{d} \eta \mathrm{d} \eta^{\prime} .
$$

Taking the minimization of Eq. (7) by the use of the Lagrange method of undetermined multipliers, the equation for minimizing spin orbitals can be obtained as follows:

$$
\begin{array}{r}
-\frac{\hbar^{2} \nabla^{2}}{2 m} \psi_{\xi}(x)+\int \mathrm{d} x^{\prime} \frac{\mu\left(\mathbf{r}, \mathbf{r}^{\prime}\right)+\mu\left(\mathbf{r}^{\prime}, \mathbf{r}\right)}{2} \sum_{\xi^{\prime}=1}^{N}\left\{\psi_{\xi^{\prime}}^{*}\left(x^{\prime}\right) \psi_{\xi^{\prime}}\left(x^{\prime}\right) \psi_{\xi}(x)-\psi_{\xi^{\prime}}^{*}\left(x^{\prime}\right) \psi_{\xi}\left(x^{\prime}\right) \psi_{\xi^{\prime}}(x)\right\} \\
=\sum_{\nu=1}^{N} \varepsilon_{\xi \nu} \psi_{\nu}(x),
\end{array}
$$

where $\mu\left(\mathbf{r}, \mathbf{r}^{\prime}\right)$ and $\varepsilon_{\xi \nu}$ are the Lagrange multipliers which respectively correspond to the restriction of Eq. (8) and orthonormality of spin orbitals. The Lagrange multiplier function $\mu\left(\mathbf{r}, \mathbf{r}^{\prime}\right)$ should be determined by requiring spin orbitals to yield a given $\gamma^{(2)}\left(\mathbf{r r}^{\prime} ; \mathbf{r r}^{\prime}\right)$. That is, $\mu\left(\mathbf{r}, \mathbf{r}^{\prime}\right)$ can be written as $\mu\left(\mathbf{r}, \mathbf{r}^{\prime}\right)=\mu\left[\gamma^{(2)}\right]\left(\mathbf{r}, \mathbf{r}^{\prime}\right)$.

Using $F\left[\gamma^{(2)}\right]$ and $T_{s}\left[\gamma^{(2)}\right]$, the exchange-correlation energy functional $E_{x c}\left[\gamma^{(2)}\right]$ is defined as

$$
F\left[\gamma^{(2)}\right]=T_{s}\left[\gamma^{(2)}\right]+U\left[\gamma^{(2)}\right]+E_{x c}\left[\gamma^{(2)}\right]
$$

where $U\left[\gamma^{(2)}\right]$ is the usual Hartree term. Here we need the note on Eq. (10). The domain of $F\left[\gamma^{(2)}\right]$ is $N$-representable PD's, that is the set $C$. On the other hand, $T_{s}\left[\gamma^{(2)}\right]$ is defined with reference to PD's which belong to the set $C^{\prime}$. 
Substituting Eq. (10) into Eq. (5) and taking the variation with respect to the PD, we can get $\mu\left[\tilde{\gamma}_{0}^{(2)}\right]\left(\mathbf{r}, \mathbf{r}^{\prime}\right)$ of Eq. (9), which reproduces $\tilde{\gamma}_{0}^{(2)}\left(\mathbf{r r}^{\prime} ; \mathbf{r r}^{\prime}\right)$ :

$$
\mu\left[\tilde{\gamma}_{0}^{(2)}\right]\left(\mathbf{r}, \mathbf{r}^{\prime}\right)=\left.\frac{\delta U\left[\gamma^{(2)}\right]}{\delta \gamma^{(2)}\left(\mathbf{r r}^{\prime} ; \mathbf{r r}^{\prime}\right)}\right|_{\gamma^{(2)}=\tilde{\gamma}_{0}^{(2)}}+\left.\frac{\delta E_{x c}\left[\gamma^{(2)}\right]}{\delta \gamma^{(2)}\left(\mathbf{r} \mathbf{r}^{\prime} ; \mathbf{r r}^{\prime}\right)}\right|_{\gamma^{(2)}=\tilde{\gamma}_{0}^{(2)}}+\frac{2}{N-1} v_{e x t}(\mathbf{r}),
$$

where $\tilde{\gamma}_{0}^{(2)}\left(\mathbf{r r}^{\prime} ; \mathbf{r r}^{\prime}\right)$ is the best solution within $C^{\prime}$.

Next we consider the exchange-correlation energy functional $E_{x c}\left[\gamma^{(2)}\right]$. Using Eq. (11), Eq. (3) is rewritten as

$$
F\left[\gamma^{(2)}\right]=T\left[\gamma^{(2)}\right]+e^{2} \iint \mathrm{d} \mathbf{r} \mathrm{d} \mathbf{r}^{\prime} \frac{\gamma^{(2)}\left(\mathbf{r r}^{\prime} ; \mathbf{r r}^{\prime}\right)}{\left|\mathbf{r}-\mathbf{r}^{\prime}\right|}
$$

where $T\left[\gamma^{(2)}\right]$ is defined as the expectation value of $\hat{T}$ with respect to the wave function $\Psi\left[\gamma^{(2)}\right]$. Note that $T\left[\gamma^{(2)}\right]$ coincides with the ground-state value of the real kinetic energy when $\gamma^{(2)}=\gamma_{0}^{(2)}$. Comparing Eq. (12) to Eq. (10), we get

$$
E_{x c}\left[\gamma^{(2)}\right]=\Delta T_{x c}\left[\gamma^{(2)}\right]+E_{x c}^{\prime}\left[\gamma^{(2)}\right]
$$

where the kinetic contribution of the exchange-correlation energy functional $\Delta T_{x c}\left[\gamma^{(2)}\right]$ and the authentic exchangecorrelation energy $E_{x c}^{\prime}\left[\gamma^{(2)}\right]$ are respectively given by

$$
\Delta T_{x c}\left[\gamma^{(2)}\right] \equiv T\left[\gamma^{(2)}\right]-T_{s}\left[\gamma^{(2)}\right]
$$

and

$$
E_{x c}^{\prime}\left[\gamma^{(2)}\right]=\frac{e^{2}}{2} \iint \mathrm{d} \mathbf{r} \mathrm{d} \mathbf{r}^{\prime} \frac{2 \gamma^{(2)}\left(\mathbf{r r}^{\prime} ; \mathbf{r r}^{\prime}\right)}{\left|\mathbf{r}-\mathbf{r}^{\prime}\right|}-U\left[\gamma^{(2)}\right]
$$

Equation (13) is similar to that of the conventional DFT. Here note that devising the approximate form of $E_{x c}^{\prime}\left[\gamma^{(2)}\right]$ is not needed in the present scheme since $E_{x c}^{\prime}\left[\gamma^{(2)}\right]$ is explicitly expressed as a functional of the PD.

Substitution of Eqs. (11) and (13) into Eq. (9), followed by the unitary transformation of $\varepsilon_{\mu \nu}$ and constant terms which are brought by the substitution, leads to the single-particle equation of the canonical form;

$$
\begin{aligned}
& \left\{-\frac{\hbar^{2} \nabla^{2}}{2 m}+v_{\text {ext }}(\mathbf{r})\right\} \chi_{\xi}(x) \\
& +\int \mathrm{d} x^{\prime}\left\{\frac{e^{2}}{\left|\mathbf{r}-\mathbf{r}^{\prime}\right|}+\left.\frac{1}{2} \frac{\delta \Delta T_{x c}\left[\gamma^{(2)}\right]}{\delta \gamma^{(2)}\left(\mathbf{r r}^{\prime} ; \mathbf{r} \mathbf{r}^{\prime}\right)}\right|_{\gamma^{(2)}=\tilde{\gamma}_{0}^{(2)}}+\left.\frac{1}{2} \frac{\delta \Delta T_{x c}\left[\gamma^{(2)}\right]}{\delta \gamma^{(2)}\left(\mathbf{r}^{\prime} \mathbf{r} ; \mathbf{r}^{\prime} \mathbf{r}\right)}\right|_{\gamma^{(2)}=\tilde{\gamma}_{0}^{(2)}}\right\} \\
& \quad \times \sum_{\nu=1}^{N}\left\{\chi_{\nu}^{*}\left(x^{\prime}\right) \chi_{\nu}\left(x^{\prime}\right) \chi_{\xi}(x)-\chi_{\nu}^{*}\left(x^{\prime}\right) \chi_{\xi}\left(x^{\prime}\right) \chi_{\nu}(x)\right\}=\varepsilon_{\xi} \chi_{\xi}(x),
\end{aligned}
$$

where $\chi_{\xi}(x)$ is the spin orbital which is converted from $\psi_{\xi}(x)$ via the unitary transformation. Since the SSD is generally kept invariant under the unitary transformation, the reproduced form of the PD of Eq. (8) is rewritten as

$$
\tilde{\gamma}_{0}^{(2)}\left(\mathbf{r r}^{\prime} ; \mathbf{r r}^{\prime}\right)=\frac{1}{2} \sum_{\mu, \nu=1}^{N} \iint\left\{\chi_{\mu}^{*}(x) \chi_{\nu}^{*}\left(x^{\prime}\right) \chi_{\mu}(x) \chi_{\nu}\left(x^{\prime}\right)-\chi_{\mu}^{*}(x) \chi_{\nu}^{*}\left(x^{\prime}\right) \chi_{\nu}(x) \chi_{\mu}\left(x^{\prime}\right)\right\} \mathrm{d} \eta \mathrm{d} \eta^{\prime} .
$$

Equations (16) and (17) are final expressions for the single-particle equations which should be solved self-consistently. It is free from the self-interaction like the Hartree-Fock equation.

The single-particle equation (16) is intelligible to us because it can be regarded as the modified Hartree-Fock equation to which kinetic contributions are added. If $\Delta T_{x c}\left[\gamma^{(2)}\right]$ terms are neglected, Eq. (16) exactly coincides with the Hartree-Fock equation. Due to $\Delta T_{x c}\left[\gamma^{(2)}\right]$ terms, spin orbitals deviate from solutions of the Hartree-Fock equation. Not only the additional terms concerning $\Delta T_{x c}\left[\gamma^{(2)}\right]$, but also values of the Hartree and Fock potentials are different from the Hartree-Fock equation. All of these differences come from $\Delta T_{x c}\left[\gamma^{(2)}\right]$ terms, and should be recognized as correlation effects. 
From another point of view, let us show again that $\Delta T_{x c}\left[\gamma^{(2)}\right]$ terms are the cause of correlation effects in the present scheme. Along the argument in Ref. [22], the following functional is introduced here:

$$
e^{\prime}[\Phi]=\langle\Phi|\hat{T}| \Phi\rangle+e^{2} \iint \frac{\gamma^{(2)}\left(\mathbf{r} \mathbf{r}^{\prime} ; \mathbf{r r}^{\prime}\right)}{\left|\mathbf{r}-\mathbf{r}^{\prime}\right|} \mathrm{d} \mathbf{r} \mathrm{d} \mathbf{r}^{\prime}+\frac{2}{N-1} \int v_{e x t}(\mathbf{r}) \gamma^{(2)}\left(\mathbf{r} \mathbf{r}^{\prime} ; \mathbf{r} \mathbf{r}^{\prime}\right) \mathrm{d} \mathbf{r} \mathrm{d} \mathbf{r}^{\prime},
$$

where $\gamma^{(2)}\left(\mathbf{r r}^{\prime} ; \mathbf{r r}^{\prime}\right)$ is expressed as the expectation value with respect to the $\operatorname{SSD} \Phi$. $e^{\prime}[\Phi]$ can be rewritten as the expectation value of $\hat{H}$ with respect to $\Phi$. Therefore, the minimum value of $e^{\prime}[\Phi]$ is obtained at $\Phi=\Phi_{H F}$, where $\Phi_{H F}$ is the ground-state wave function of the Hartree-Fock approximation. On the other hand, we can also show that $\underset{\Phi}{\operatorname{Min}} e^{\prime}[\Phi]=\operatorname{Min}_{\gamma^{(2)} \in C^{\prime}}\left\{E\left[\gamma^{(2)}\right]-\Delta T_{x c}\left[\gamma^{(2)}\right]\right\}$ similarly to the proof of Eq. (4-12) of Ref. [22]. Therefore, we get

$$
\left\langle\Phi_{H F}|\hat{H}| \Phi_{H F}\right\rangle=\operatorname{Min}_{\gamma^{(2)} \in C^{\prime}}\left\{E\left[\gamma^{(2)}\right]-\Delta T_{x c}\left[\gamma^{(2)}\right]\right\}
$$

This relation indicates that the ground-state energy which is expressed as $\underset{\gamma^{(2)} \in C^{\prime}}{\operatorname{Min}} E\left[\gamma^{(2)}\right]$ in our scheme is different from that calculated in the Hartree-Fock approximation. The difference originates from $\Delta T_{x c}\left[\gamma^{(2)}\right]$. Since $\Delta T_{x c}\left[\gamma^{(2)}\right]$ is rewritten as $\operatorname{Min}_{\Psi \rightarrow \gamma^{(2)}}\langle\Psi|\hat{T}| \Psi\rangle-\operatorname{Min}_{\Phi \rightarrow \gamma^{(2)}}\langle\Phi|\hat{T}| \Phi\rangle[25]$, it holds the inequality $\Delta T_{x c}\left[\gamma^{(2)}\right] \leq 0$. Therefore, it is expected that the ground-state energy becomes lower than $\left\langle\Phi_{H F}|\hat{H}| \Phi_{H F}\right\rangle$. That is to say, correlation effects are incorporated into the present scheme if $\Delta T_{x c}\left[\gamma^{(2)}\right]$ is taken into account.

The negative value of $\Delta T_{x c}\left[\gamma^{(2)}\right]$ also leads to the inequality $T_{s}\left[\gamma^{(2)}\right] \geq T_{H F}[26]$, where $T_{H F}$ is the kinetic energy within the Hartree-Fock approximation. This means again that correlation effects are incorporated into the singleparticle equation through $\Delta T_{x c}\left[\gamma^{(2)}\right]$ because correlation effects have a tendency to raise the kinetic energy. In addition, we shall give a comment on the possibility of $\Delta T_{x c}\left[\gamma^{(2)}\right]=0$. If the wave functions which yield the PD of $C^{\prime}$ were SSD's alone, $\Delta T_{x c}\left[\gamma^{(2)}\right]$ would be always zero. However, that is not the case. Indeed, there exists the set of wave functions which give the $\mathrm{PD}$ of $C^{\prime}$ and are not expressed by a $\operatorname{SSD}[19]$. Therefore, it is not the case that $\Delta T_{x c}\left[\gamma^{(2)}\right]$ is always zero.

In order to make it more feasible to perform the actual calculation of the present scheme, we propose the approximate form of $\Delta T_{x c}\left[\gamma^{(2)}\right]$ by using the scaling relation of $\Delta T_{x c}\left[\gamma^{(2)}\right]$ as a sum rule. The scaling relation of $\Delta T_{x c}\left[\gamma^{(2)}\right]$ can be obtained from those of $T\left[\gamma^{(2)}\right]$ and $T_{s}\left[\gamma^{(2)}\right]$. As for the scaling relation of $T\left[\gamma^{(2)}\right]$, Levy and Ziesche[27] has derived the relation $T\left[\gamma_{\zeta}^{(2)}\right]=\zeta^{2} T\left[\gamma^{(2)}\right]$. Here $\gamma_{\zeta}^{(2)}\left(\mathbf{r r}^{\prime} ; \mathbf{r r}^{\prime}\right)$ denotes the expectation value of $\hat{\gamma}^{(2)}\left(\mathbf{r r}^{\prime} ; \mathbf{r r}^{\prime}\right)$ with respect to the scaled wave function, i.e., $\gamma_{\zeta}^{(2)}\left(\mathbf{r r}^{\prime} ; \mathbf{r r}^{\prime}\right)=\zeta^{6} \gamma^{(2)}\left(\zeta \mathbf{r} \zeta \mathbf{r}^{\prime} ; \zeta \mathbf{r} \zeta \mathbf{r}^{\prime}\right)$. In the similar way to deriving the above relation, the scaling relation of $T_{s}\left[\gamma^{(2)}\right]$ is also given by $T_{s}\left[\gamma_{\zeta}^{(2)}\right]=\zeta^{2} T_{s}\left[\gamma^{(2)}\right][28]$. Thus, we get $\Delta T_{x c}\left[\gamma_{\zeta}^{(2)}\right]=\zeta^{2} \Delta T_{x c}\left[\gamma^{(2)}\right]$. Along the technique of Ref. [29], let $\lim _{\zeta \rightarrow 1} \frac{\partial}{\partial \zeta}$ act on both sides of this relation. Utilizing the integration by parts, we obtain 30 ]

$$
\begin{aligned}
2 \Delta T_{x c}\left[\gamma^{(2)}\right]= & -\iint \gamma^{(2)}\left(\mathbf{r} \mathbf{r}^{\prime} ; \mathbf{r r}^{\prime}\right) \mathbf{r} \cdot \nabla\left\{\frac{\delta \Delta T_{x c}\left[\gamma^{(2)}\right]}{\delta \gamma^{(2)}\left(\mathbf{r} \mathbf{r}^{\prime} ; \mathbf{r r}^{\prime}\right)}\right\} \mathrm{d} \mathbf{r} \mathrm{d} \mathbf{r}^{\prime} \\
& -\iint \gamma^{(2)}\left(\mathbf{r r}^{\prime} ; \mathbf{r r}^{\prime}\right) \mathbf{r} \cdot \nabla\left\{\frac{\delta \Delta T_{x c}\left[\gamma^{(2)}\right]}{\delta \gamma^{(2)}\left(\mathbf{r}^{\prime} \mathbf{r} ; \mathbf{r}^{\prime} \mathbf{r}\right)}\right\} \mathrm{d} \mathbf{r} \mathrm{d} \mathbf{r}^{\prime}
\end{aligned}
$$

We shall use this equation as a sum rule for $\Delta T_{x c}\left[\gamma^{(2)}\right]$.

With reference to the local density approximation of the conventional DFT [2], the following form is assumed for $\Delta T_{x c}\left[\gamma^{(2)}\right]:$

$$
\Delta T_{x c}\left[\gamma^{(2)}\right]=\left.\iint \Delta t_{x c}\left(\gamma^{(2)}\right)\right|_{\gamma^{(2)}=\gamma^{(2)}\left(\mathbf{r r}^{\prime} ; \mathbf{r r}^{\prime}\right)} \mathrm{d} \mathbf{r} \mathrm{d} \mathbf{r}^{\prime}
$$

where $\Delta t_{x c}\left(\gamma^{(2)}\right)$ is an ordinary function of $\gamma^{(2)}$. Substituting Eq. (21) into Eq. (20), and neglecting the surface integral at infinity, we get the differential equation with respect to $\Delta t_{x c}\left(\gamma^{(2)}\right)$ as the necessary condition:

$$
4 \Delta t_{x c}\left(\gamma^{(2)}\right)-3 \gamma^{(2)} \frac{\partial \Delta t_{x c}\left(\gamma^{(2)}\right)}{\partial \gamma^{(2)}}=0
$$

A similar relation for $\Delta t_{x c}(\rho)$ has been derived by Levy and Perdew in the conventional DFT 31]. Solving the differential equation (22), and substituting the solution into Eq. (21), we finally get the approximate form of $\Delta T_{x c}\left[\gamma^{(2)}\right]$;

$$
\Delta T_{x c}\left[\gamma^{(2)}\right]=K \iint \gamma^{(2)}\left(\mathbf{r r}^{\prime} ; \mathbf{r r}^{\prime}\right)^{\frac{4}{3}} \mathrm{~d} \mathbf{r} \mathrm{d} \mathbf{r}^{\prime}
$$


where $K$ is the arbitrary constant. The constant $K$ may be available as an adjustable parameter in our scheme. Namely, $K$ may be determined corresponding to the magnitude of correlation effects of the system. For example, in case of atomic structures, we may fix the value by fitting total energies to those of more accurate calculations 32 .

In conclusion, this paper is aimed at searching the best solution of the PD within the set of $C^{\prime}$. The biggest merit of our theory is that the solution is physically reasonable. Namely, our solution is necessarily $N$-representable. The solution even in the set $C^{\prime}$ can contain the correlation effects through $\Delta T_{x c}$ term more or less but definitely. This point is also important. It seems worthwhile to perform the actual calculation so as to check how much the present scheme can reflect correlation effects beyond the Hartree-Fock. This is the next step to be done 32 .

[1] P. Hohenberg and W. Kohn, Phys. Rev. 136, B864 (1964).

[2] W. Kohn and L. J. Sham, Phys. Rev. 140, A1133 (1965).

[3] R. G. Parr and W. Yang, Density-Functional Theory of Atoms and Molecules (Oxford University Press, New York, 1989) Chap. 2.

[4] Many-electron densities and reduced density matrices, edited by J. Closlowski (Kluwer Academic/Plenum Publishers, New York, 2000).

[5] Concerning an attempt combining the density matrix functional theory and the model Hamiltonian, see, K. Kusakabe, cond-mat/0505703

[6] P. Ziesche, Phys. Lett. A 195, 213 (1994).

[7] P. Ziesche, Int. J. Quantum Chem. 60, 1361 (1996).

[8] A. Gonis, T. C. Schulthess, J. van Ek and P. E. A. Turchi, Phys. Rev. Lett. 77, 2981 (1996).

[9] A. Gonis, T. C. Schulthess, P. E. A. Turchi and J. van Ek, Phys. Rev. B 56, 9335 (1997).

[10] A. Nagy, Phys. Rev. A 66, 022505 (2002).

[11] A. Nagy and C. Amovilli, J. Chem. Phys. 121, 6640 (2004).

[12] P. W. Ayers, J. Math. Phys. 46, 062107 (2005).

[13] A. J. Coleman, The Force Concept in Chemistry, edited by B. M. Deb (Van Nostrand Reinhold, New York, 1981) p.418.

[14] A. J. Coleman, Rev. Mod. Phys. 35, 668 (1963).

[15] A. J. Coleman and V. I. Yukalov, Reduced Density Matrices: Coulson's Challenge (Springer-Verlag, Berlin, 2000).

[16] The fundamentals of electron density, density matrix and density functional theory in atoms, molecules and the solid states, edited by N. I. Gidopoulos and S. Wilson (Kluwer Academic Press, New York, 2003).

[17] E. R. Davidson, Chem. Phys. Lett. 246, 209 (1995).

[18] S. Kh. Samvelyan, Int. J. Quantum Chem. 65, 127 (1997).

[19] M.-E. Pistol, Chem. Phys. Lett. 400, 548 (2004).

[20] M. Levy, Proc. Natl. Acad. Sci. USA 76, 6062 (1979).

[21] E. H. Lieb, Int. J. Quant. Chem. 24, 243 (1983).

[22] M. Higuchi and K. Higuchi, Phys. Rev. B 69, 035113 (2004).

[23] K. Higuchi and M. Higuchi, Phys. Rev. B 69, 165118 (2004).

[24] A. Szabo and N. S. Ostlund, Modern Quantum Chemistry (Dover Publications, NY, 1996).

[25] By using the definition of $T\left[\gamma^{(2)}\right]$, we can get

$$
\begin{aligned}
& \operatorname{Min}_{\Psi \rightarrow \gamma^{(2)}}\langle\Psi|\hat{T}| \Psi\rangle=\operatorname{Min}_{\Psi \rightarrow \gamma^{(2)}}\{\langle\Psi|\hat{T}+\hat{W}| \Psi\rangle-\langle\Psi|\hat{W}| \Psi\rangle\} \\
& \quad=F\left[\gamma^{(2)}\right]-e^{2} \iint \frac{\gamma^{(2)}\left(\mathbf{r r}^{\prime} ; \mathbf{r r}^{\prime}\right)}{\left|\mathbf{r}-\mathbf{r}^{\prime}\right|} \mathrm{d} \mathbf{r} \mathrm{d} \mathbf{r}^{\prime}=T\left[\gamma^{(2)}\right]
\end{aligned}
$$

[26] The correlation effects likely raise the kinetic energy, i.e., $T\left[\gamma^{(2)}\right] \geq T_{H F}$. Since $\Delta T_{x c}\left[\gamma^{(2)}\right] \leq 0$, we obtain the inequality $T_{s}\left[\gamma^{(2)}\right] \geq T_{H F}$.

[27] M. Levy and P. Ziesche, J. Chem. Phys. 115, 9110 (2001).

[28] K. Higuchi and M. Higuchi, Phys. Rev. B 71, 035116 (2005).

[29] S. Erhard and E. K. U. Gross, Phys. Rev. A 53, R5 (1996).

[30] It is easily confirmed that the same relation as Eq. 20] can be obtained for $T\left[\gamma^{(2)}\right]$ by employing the virial theorem. As for the virial theorem, see, Ref. [28].

[31] M. Levy and J. P. Perdew, Phys. Rev. A 32, 2010 (1985).

[32] K. Higuchi and M. Higuchi, in preparation. 